\title{
The Impact of Inhaled Salbutamol on Repeated Sprint Ability in Pre- Fatigued Soccer Players
}

\author{
Dickinson $\mathrm{J}^{1}$, $\mathrm{Hu} \mathrm{J}^{2}$, Chester $\mathrm{N}^{2}$, Loosemore $\mathbf{M}^{3}$, and Whyte $\mathbf{G}^{2}$ \\ ${ }^{1}$ Endurance Research Group, University of Kent, Chatham Maritime, UK \\ ${ }^{2}$ Research Institute for Sport and Exercise Sciences, Liverpool John Moores University, Liverpool, UK \\ ${ }^{3}$ The Institute of Sport, Exercise and Health, University College London, London, UK
}

\author{
Abstract \\ Objectives: Investigate the ergogenic effect of inhaling up to $1600 \mu \mathrm{g}$ of salbutamol on intermittent running \\ performance in pre-fatigued soccer players.
}

Methods: In a single blind randomised repeated measures design seven male and six female soccer players volunteered to participant. All participants were regularly playing competitive soccer and had no history of asthma. Following familiarisation sessions participants visited the exercise physiology laboratory on three occasions to complete an intermittent running protocol followed by twelve $17.5 \mathrm{~m}$ sprints. Prior to each trial participants inhaled either: placebo, $800 \mu \mathrm{g}$ inhaled salbutamol (SAL800) or $1600 \mu \mathrm{g}$ inhaled salbutamol (SAL1600). Following completion of the sprints a sample from the first urine passed was analysed for salbutamol concentration. A repeated measures ANOVA was used to compare the mean sprint time, maximal sprint power, peak blood lactate post sprints and post sprint salbutamol urine concentration between conditions.

Results: Mean sprint time, maximum power, maximum velocity, peak HR and peak blood lactate during the 17.5 $\mathrm{m}$ sprints were not significantly different between treatments in soccer players. There was no significant difference between male and female players in urine drug concentration following SAL800 (mean + SD; $201.47+294.47 \mathrm{ng}^{-\mathrm{ml}^{-1}}$ vs. $\left.180.2+102.15 \mathrm{ng} \mathrm{ml}^{-1}\right)$ or SAL1600 $\left(739.24+549.21 \mathrm{ng} \cdot \mathrm{ml}^{-1} \mathrm{vs.} 879.58+633.14 \mathrm{ng} \cdot \mathrm{ml}^{-1}\right)$. Three players urine drug concentrations were above the WADA decision limit set at $1200 \mathrm{ng}^{-\mathrm{ml}^{-1}}$

Conclusions: Inhaling up to $1600 \mu \mathrm{g}$ inhaled salbutamol did not significantly improve repeated sprint performance. However, inhalation of $1600 \mu \mathrm{g}$ may result in a urine concentration above the current WADA upper limit and decision limit leading to a positive test. Athletes should ensure they use inhaled salbutamol at therapeutic doses to avoid the risk of breaching the WADA decision limit.

Keywords: Anti-doping; WADA code; Asthma; Treatment; Athlete care; Abuse; Adverse analytical finding

\section{Introduction}

Between 2002 and 2010, International Olympic Committee (IOC) established the requirement that athletes present evidence of current asthma or exercise induced bronchoconstriction (EIB) through the Therapeutic Use Exemption (TUE) process. The weight of evidence supports the improved health care of athletes following the introduction of the TUE $[1,2]$. In contrast, there is limited evidence to suggest inhaled doses of $\beta_{2}$-agonist have significant ergogenic effects according to a study by Pluim et al. The current List of banned substances and methods allows athletes to inhale up to $1600 \mu \mathrm{g}$ salbutamol without the requirement of a TUE [3].

To date, there are no published studies examining the impact of inhaled short acting $\beta_{2}$-agonists on team game performance. Whilst athletes are usually prescribed $200-400 \mu \mathrm{g}$ of inhaled salbutamol, our experience suggests that athletes are often instructed to take salbutamol on an 'as needed basis' that could be interpreted as an ability to inhale an unlimited amount of salbutamol to combat respiratory symptoms. Given the global importance of team games such as Soccer it is imperative that the performance impact of inhaled short acting $\beta_{2}$ agonists be examined to justify their position on the WADA list of prohibited substances.

The purpose of this study was to examine the ergogenic effect of inhaling $800 \mu \mathrm{g}$ and $1600 \mu \mathrm{g}$ of salbutamol versus placebo repeated sprint performance in pre-fatigued soccer players. Furthermore, to investigate the impact of prior exercise and gender on urine salbutamol concentrations following inhalation of the WADA recommended upper limit of $1600 \mu \mathrm{g}$ of Salbutamol.

\section{Materials and Method}

Ethical approval for this study was provided by the Liverpool John Moores University Local Ethics Committee (ethics no: 09E18GW). Seven male (mean + SD; age $23.1+3.9$ years; weight $72.9+4.3 \mathrm{~kg}$; height $177.0+4.7 \mathrm{~cm})$ and six female $(21.3+1.4$ years; weight $63.9+$ $5.8 \mathrm{~kg}$; height $162.3+4.7 \mathrm{~cm}$ ) soccer players volunteered and provided written and verbal informed consent. All players played regular competitive 11 a-side soccer at least once a week and trained specifically for soccer at least twice a week. All participants were free from asthma and EIB. This was confirmed by the presentation of a negative Eucapnic Voluntary Hyperpnoea challenge [4]. On three separate occasions players were required to complete a 52 minute intermittent running

*Corresponding author: Dr. John Dickinson, School of Sport and Exercise Sciences, University of Kent, Medway Building, Chatham Maritime, Kent, ME4 4AG, UK, Tel: 0044 (0) 1634 202998; Fax: 0044 (0) 1634 888890, E-mail: J.W.Dickinson@kent.ac.uk

Received July 30, 2015; Accepted August 26, 2015; Published August 31, 2015

Citation: Dickinson J, Hu J, Chester N, Loosemore M, Whyte G (2015) The Impact of Inhaled Salbutamol on Repeated Sprint Ability in Pre-Fatigued Soccer Players. J Sports Med Doping Stud 5: 164. doi:10.4172/2161-0673.1000164

Copyright: @ 2015 John D, et al. This is an open-access article distributed under the terms of the Creative Commons Attribution License, which permits unrestricted use, distribution, and reproduction in any medium, provided the original author and source are credited. 
protocol followed by twelve, $17.5 \mathrm{~m}$ sprints. Each trial was completed in a temperate environment $\left(18^{\circ} \mathrm{C}\right.$ and $40 \%$ relative humidity). Each trial was separated by 7 days. Fifteen minutes prior to the initiation of each intermittent run players inhaled one of the following treatments, via a pocket chamber, in a randomised single blind design:

Treatment 1: 16 inhalations of placebo (PLA)

Treatment 2: 8 inhalations of $100 \mu \mathrm{g}$ salbutamol, 8 inhalation of placebo (SAL800)

Treatment 3: 16 inhalations of $100 \mu \mathrm{g}$ salbutamol (SAL1600)

Maximal flow volume loops were recorded at baseline, 10 minutes post-treatment inhalation and 5 minutes post-simulated soccer match. A $90 \mathrm{ml}$ urine sample was obtained between 30 and 180 minutes following the cessation of the twelve $17.5 \mathrm{~m}$ sprints.

The intermittent running protocol involved players completing 52 minutes of an adapted version of a validated soccer game performance treadmill test $[5,6]$. The test was conducted on a motorised treadmill (Pulsar, h/p/cosmos, Germany). The players were asked to complete five LOW and two HIGH intensity stages (Figure 1). The female low and high intensity stages were $10 \%$ slower than the male speeds. The stages were completed in the following order LOW, LOW, HIGH, LOW, LOW, HIGH, LOW. Between each stage there was a one minute stationary period (Figure 1).

Fifteen minutes following the soccer specific intermittent running protocol participants completed twelve $17.5 \mathrm{~m}$ sprints with a $10 \mathrm{~s}$ recovery between each sprint. This test is an adaption of the Repeated Anaerobic Sprint Test (RAST), which involves $6 \times 35 \mathrm{~m}$ sprints [7]. The players completed the $17.5 \mathrm{~m}$ sprints on a non-motorised treadmill (Force Treadmill System, Woodway, SA). For each sprint the following variables were recorded: time, peak power and peak velocity. HR was monitored during the sprints and peak HR across all the twelve sprints was recorded. Consistent verbal encouragement was given to the players during each sprint. Players were encouraged to complete the sprints as fast as they could.

Three maximal flow-volume loops were measured at baseline, ten minutes after inhalation of PLA, SAL800 and SAL1600, and five minutes after the soccer specific intermittent running protocol. On each occasion maximal flow volumes were measured according to the European Respiratory Society (ERS) criteria [8].

Prior to the commencement of each trial players were asked to provide a urine sample in order to void them of urine, after which the placebo or salbutamol was administered as described above. Players were instructed to collect the first sample of urine passed following completion of the laboratory-based tests in line with in-competition anti-doping procedures as outlined in the World Anti-Doping Code International Standard for Testing [9]. Consumption of water during this period was encouraged ad libitum to ensure diuresis. From the sample provided by each subject a $20 \mathrm{ml}$ aliquot of urine was stored at $-80^{\circ} \mathrm{C}$ until analysis.

All urinalysis was performed at HFL Sport Science (Fordham, UK) an independent drug surveillance laboratory and former WADAaccredited laboratory. Sample preparation involved the addition of 200 ng of Salbutamol-D3 (NMI) as an internal standard to $1 \mathrm{ml}$ of urine. Following the addition of $2 \mathrm{ml}$ of $0.1 \mathrm{M}$ phosphate buffer $\mathrm{pH} 6.8$ and $100 \mu \mathrm{l}$ of $E$. Coli enzyme ( $\beta$-glucuronidase) solution the mixture was incubated overnight at $37^{\circ} \mathrm{C}$. Strata XC $60 \mathrm{mg}$ solid phase extraction cartridges (Phenomenex, Macclesfield, UK) were conditioned with 3 $\mathrm{ml}$ of methanol followed by $3 \mathrm{ml}$ of reagent grade water. Following centrifugation at $3500 \mathrm{rpm}$ for $5 \mathrm{~min}$ the samples were applied to the cartridges. The cartridges were then washed with $3 \mathrm{ml}$ of $0.1 \mathrm{M}$ acetate buffer $\mathrm{pH} 9.0$ followed by $3 \mathrm{ml}$ of reagent grade water, $3 \mathrm{ml}$ of $0.1 \mathrm{M}$ $\mathrm{HCl}, 3 \mathrm{ml}$ of methanol and $3 \mathrm{ml}$ of diethyl ether. The cartridges were then dried for $5 \mathrm{~min}$ for vacuum and samples were eluted into glass vials with two, $1 \mathrm{ml}$ of basic drug elution solvent ( $160 \mathrm{ml}$ ethyl acetate, $34 \mathrm{ml}$ propan-2-ol and $6 \mathrm{ml} \mathrm{34 \%} \mathrm{ammonia} \mathrm{solution).} \mathrm{Samples} \mathrm{were}$ then evaporated to dryness at ambient temperature using a centrifugal vacuum concentrator (Genevac Ltd, Ipswich, UK) and reconstituted in $10 \mu \mathrm{l}$ of isopropanol followed by $200 \mu \mathrm{l}$ of basic reconstitution solution (495 ml of 0.1 acetic acid mixed with $5 \mathrm{ml}$ Benzyldimethylphenyl Ammonium). Samples were centrifuged at $3000 \mathrm{rpm}$ for $10 \mathrm{~min}$ prior to LCMS submission. Samples were injected onto a Thermo Scientific Accela HPLC system coupled to a Thermo Scientific LTQ Orbitrap Discovery Mass Spectrometer (Thermo Fisher Scientific, Waltham, USA). Chromatographic separation was performed on a Waters Atlantis T3 column $(2.1 \times 100 \mathrm{~mm}$, particle size $3 \mu \mathrm{m}$; Waters Ltd, Elstree, $\mathrm{UK}$ ) at $35^{\circ} \mathrm{C}$. The mobile phase was a gradient system of $0.1 \%$ acetic acid aqueous solution containing uracil (300 ng. $\left.\mathrm{ml}^{-1}\right)$ and $0.1 \%$ acetic acid in acetonitrile containing uracil $\left(300 \mathrm{ng} \cdot \mathrm{ml}^{-1}\right)$ set at a flow rate of $0.4 \mathrm{ml} \cdot \mathrm{min}^{-1}$.

The urine salbutamol concentrations reported correspond to the sum of the free and glucuronide conjugates. The samples were analysed over the calibration range of 10-2000 $\mathrm{ng} \cdot \mathrm{ml}^{-1}$. Samples with salbutamol concentrations greater than the upper limit of quantification were diluted with blank human urine prior to analysis. The lower limit of
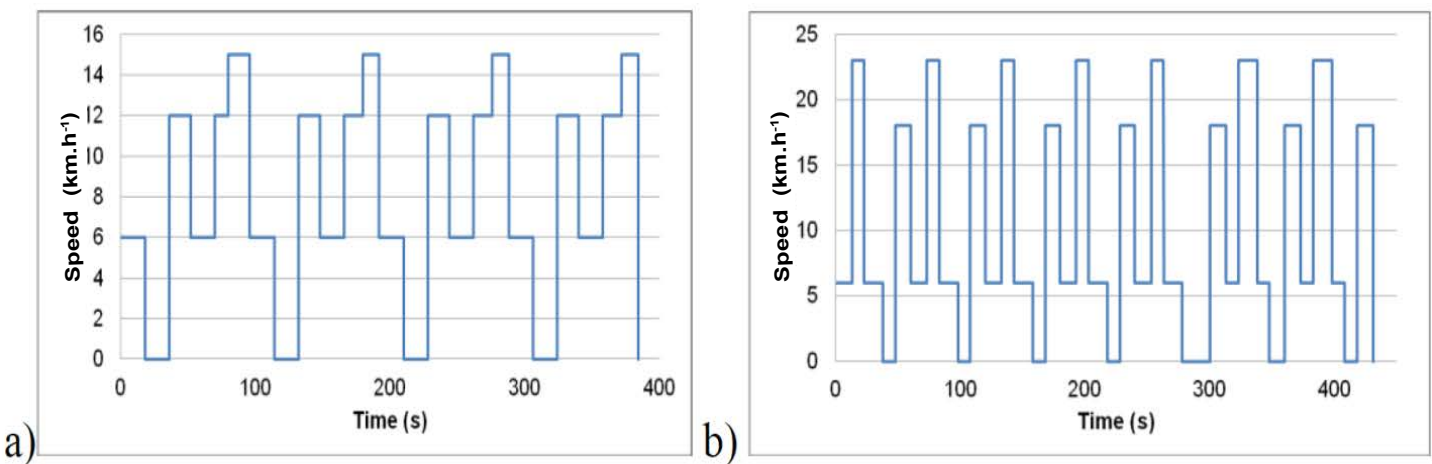

Figure 1: Low (a) and high (b) intensity phases of the male soccer specific treadmill run. 
quantification was accepted as the lowest standard on the calibration curve (10 ng. $\left.\mathrm{ml}^{-1}\right)$.

Male and female data were analysed together. Mean sprint time to complete the twelve, $17.5 \mathrm{~m}$ sprints under each of the treatments was analysed by a repeated measures ANOVA. Similar analyses were undertaken for peak power, peak velocity and peak HR. Repeated measures ANOVA were used to compare FEV1, FVC, PEF and FEF2575. A p value of $<0.05$ was deemed significant for all analysis.

\section{Results}

All seven male and six female players completed the football specific run and twelve, $17.5 \mathrm{~m}$ sprints under each condition. No participant reported any adverse symptoms from inhaling either 800 or $1600 \mu \mathrm{g}$ salbutamol.Under each of the three treatments no significant difference was observed in mean sprint time (PLA 4.74+0.65s; SAL800 4.65+0.55 s; SAL1600 4.74+0.53 s; $\mathrm{p}=0.35$ ), peak power (PLA 1641.7+492.0 W; SAL800 1681.7+524.0 W; SAL1600 1738.8+499.9 W; p=0.40), peak velocity (PLA 4.99+0.66 m.s ${ }^{-1}$; SAL800 5.00+0.70 m.s ${ }^{-1}$; SAL1600 $5.01+0.68 \mathrm{~m} . \mathrm{s}^{-1} ; \mathrm{p}=0.51$ ), peak HR (PLA 182.6+5.1 b.min ${ }^{-1}$; SAL800 $184.6+4.5$ b. $\mathrm{min}^{-1}$; SL1600 $183.4+4.54$ b. $\mathrm{min}^{-1}$; $\mathrm{p}=0.22$ ) or, peak lactate (PLA 11.55+2.16 mmol. $^{-1}$; SAL800 $12.45+2.59$ mmol. $^{-1}$; $13.52+2.3$ mmol..$\left.^{-1} ; \mathrm{p}=0.06\right)$.

Maximal lung function did not significantly alter between treatments at baseline, 10 minutes after inhalation of treatment or 5 minutes after the completion of the football specific run.

Following inhalation of $800 \mu \mathrm{g}$ and $1600 \mu \mathrm{g}$ of salbutamol and completion of a football-specific running protocol and twelve, $17.5 \mathrm{~m}$ sprints under temperate $\left(20^{\circ} \mathrm{C}, 40 \% \mathrm{RH}\right)$ ambient conditions the cohort reported mean $( \pm \mathrm{SD})$ urine drug concentrations of $250.91+234.51$ ng. $\mathrm{ml}^{-1}$ and $804.02+568.46 \mathrm{ng} \cdot \mathrm{ml}^{-1}$, respectively (see figure 2). Whilst mean $(+\mathrm{SD})$ urine drug concentrations following inhalation of 800 $\mu \mathrm{g}$ of salbutamol was $301.47+294.47 \mathrm{ng} \cdot \mathrm{ml}^{-1}$ in males compared to $180.12+102.15 \mathrm{ng} \cdot \mathrm{ml}^{-1}$ in females there was no significant difference according to sex (Figure 2). Likewise, there were no significant sex differences in urine drug concentrations following inhalation of 1600 $\mu \mathrm{g}$ of salbutamol in male $739.24+549.21 \mathrm{ng} \cdot \mathrm{ml}^{-1}$ and female $879.58+$ 633.14 ng. $\mathrm{ml}^{-1}$ players. Nevertheless there was a high degree of interindividual differences in urine drug concentrations amongst male and female participants. Whilst mean urine drug concentrations did not breach the WADA urinary threshold for salbutamol two male and three female players recorded urine drug concentrations that exceeded the threshold (1000 ng. $\mathrm{ml}^{-1}$ ), of which, three cases were above the
WADA decision limit set at $1200 \mathrm{ng} \cdot \mathrm{ml}^{-1}$.

\section{Discussion}

The results from the current study suggest that inhaling up to 1600 $\mu \mathrm{g}$ of salbutamol does not result in an improvement in repeated sprint performance in pre-fatigued soccer players. Despite this, our results demonstrate that it is possible for athletes to present with a salbutamol urine concentration above $1000 \mathrm{ng}$.ml-1 following inhalation of up to $1600 \mu \mathrm{g}$ of salbutamol.

Previous studies focused on inhaled salbutamol have generally employed non-specific performance trials such as running to exhaustion or physiological markers of performance [10]. Few of the previous studies have demonstrated any improvement following the inhalation of acute doses of up to $800 \mu \mathrm{g}$ Salbutamol on running time to exhaustion, $\mathrm{O} 2 \mathrm{max}$, peak power, $20 \mathrm{~km}$ cycling time-trial and total work during a $30 \mathrm{~s}$ Wingate test [10]. Dickinson et al. [11] reported no improvement in $5 \mathrm{~km}$ running time trial performance following inhalation of $1600 \mu \mathrm{g}$ salbutamol. Elers et al. [12] suggested inhaling an acute dose of up to $4000 \mu \mathrm{g}$ salbutamol resulted in no improvement in cycling time to exhaustion or oxygen kinetics. Accordingly, from a performance perspective the current WADA guidelines permitting athletes to inhale up to $1600 \mu \mathrm{g}$ in a single day appear appropriate as there is no resultant improvement in performance in non-asthmatic athletes. Furthermore, there also appears to be no improvement in strength, power or endurance when none asthmatic athletes inhale 1600 $\mu \mathrm{g}$ Salbutamol on a daily basis for 6 weeks [13]. However, in contrast to our findings a recent study has suggest there may be an improvement in swimming sprint performance and maximal contraction of the quadriceps femoris if inhaled salbutamol is taken in combination with inhaled long acting $\beta 2$-agonists [14]. In addition, oral salbutamol taken acutely and over a two week period has been shown to improve peak power output on repeated cycling sprint performance [15]. However, Hostrup et al. [15] did report $\beta 2$-adrenergic stimulation was blunted in the second and third sprints. In our study we used a soccer specific running protocol to induce fatigue in our participants prior to the $17.5 \mathrm{~m}$ sprints. It may be possible that the preceding soccer specific treadmill run impacted on the potential for inhaled salbutamol to improve individual sprint and repeated sprint performance. Future studies investigating repeated sprint performance may benefit from inhaling salbutamol directly before the sprinting protocol.

The current WADA regulations impose a urinary salbutamol threshold of 1000 ng.ml-1for salbutamol [3]. This regulation is to distinguish legitimate therapeutic use from misuse. Supporting such a threshold are maximal dosing recommendations of $1600 \mu \mathrm{g}$ per day
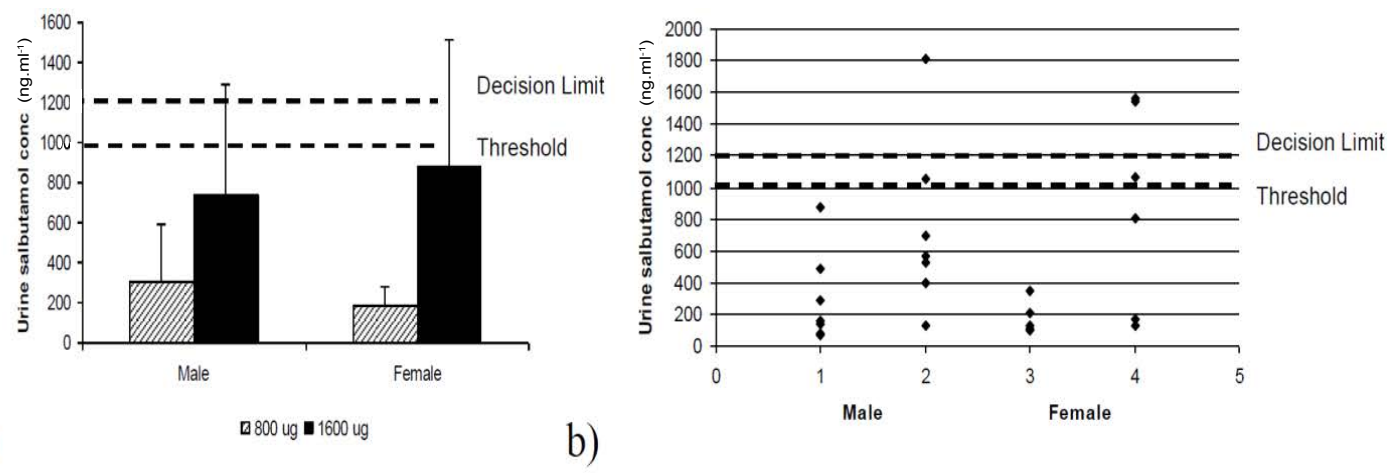

a)

Figure 2: (a) Mean (+ SD) and (b) Individual urine SAL concentration
inhalation of $800 \mu \mathrm{g}$ or $1600 \mu \mathrm{g}$ SAL in male and female players. 
[3]. However, whilst the recommended dosing regimen is between 100 and $400 \mu \mathrm{g}$ up to four times daily, salbutamol is typically prescribed pro re nata ('when required'). Individuals encouraged to administer salbutamol pro re nata may inhale doses of salbutamol over and above such recommendations for therapeutic purposes, either intentionally or inadvertently. In such cases athletes are at risk of exceeding the WADA threshold and in doing so, facing possible anti-doping sanctions.

Previous research has attempted to examine the excretion of salbutamol administered by different routes including oral and inhaled $[16,17]$ and across a range of acute doses from $200 \mu \mathrm{g}$ and $800 \mu \mathrm{g}[18]$ and repeated doses over several hours [19]. The current study is the first to examine the urinary excretion of an acute, high dose in comparison with an intermediate dose following intermittent running in soccer players. Whilst the findings did not suggest any differences according to gender there was a large variation between individuals at the various doses. This large inter-subject variation confirmed the likelihood that individuals inhaling acute, high doses of salbutamol may exceed the current WADA urinary threshold of $1000 \mathrm{ng} \cdot \mathrm{ml}^{-1}$. Indeed the current study also demonstrates that following such a dosing regimen may result in urine salbutamol levels in excess of $1200 \mathrm{ng} \cdot \mathrm{ml}^{-1}$. This concentration is significant since it represents a level referred to by WADA as the 'Decision Limit, above which a sample would be classified as an adverse analytical finding (AAF) [9] and warrant further investigation.

The maximal recommended dosing regimen for salbutamol clearly acts as an upper limit for those requiring immediate relief of asthma symptoms. Individuals who regularly use high doses of salbutamol or exceed these recommendations clearly demonstrate poor management of their condition. There lies the problem from an anti-doping regulatory perspective when the evidence to support ergogenic properties is not present and the likelihood of poor control of asthma and EIB are high. From a sports medicine perspective improved care may constitute better diagnosis, management and education amongst athletes. Regardless of whether salbutamol is on or off the WADA list of banned substances team medical officers must follow best practice in the diagnosis of respiratory conditions such as EIB prior to providing inhalers for therapeutic use [20].

\section{Conclusion}

Our findings suggest there is no ergogenic benefit, from inhaling up to $1600 \mu \mathrm{g}$ of salbutamol, on intermittent running performance in soccer players. However, inhalation of $1600 \mu \mathrm{g}$ may result in a urine concentration above the current WADA upper limit and decision limit leading to a positive test finding. Athletes should ensure they use inhaled salbutamol at therapeutic doses to avoid the risk of breaching the WADA decision limit.

\section{Conflict of Interest}

None to declare

\section{Discloser of Funding}

This projected was funded by the World Anti-Doping Agency (WADA).

\section{Acknowledgments}

The authors would like to thank the technical support of Rob Allan and Andrew Hulton during the data collection phase of this study.

\section{References}

1. Dickinson J, Whyte G, McConnell A, Harries M (2005) Impact of change in the IOC-MC asthma criteria: a British perspective. Thorax 60: 629-632.

2. Parsons J, Kaeding C, Phillips G, Jarjoura D, Wadley G, et al. (2007) Prevalence of exercise-induced bronchospasm in a cohort of varsity college athletes. Med Sci Sports Exerc 39: 1487-1492.
3. The World Anti-Doping Agency (2015) The 2015 Prohibited List International Standard.

4. Anderson S, Argyros G, Magnussen H, Holzer K (2001) Provocation by eucapnic voluntary hyperpnoea to identify exercise induced bronchoconstriction. $\mathrm{Br} \mathrm{J}$ Sports Med 35: 344-347.

5. Drust B, Reilly T, Cable T (2000) Physiological responses to laboratory-based soccer-specific intermittent and continuous exercise. J Sports Sci 18: 885-892.

6. Drust B, Atkinson G, Reilly $T$ (2007) Future perspectives in the evaluation of the physiological demands of soccer. Sports Med 37: 783-805.

7. Draper N, Whyte G (1997) Here's a new running based test of anaerobic performance for which you need only a stopwatch and a calculator. Peak Performance 97: 3-5

8. Miller M, Hankinson J, Brusasco V, Burgos F, Casaburi R, et al. (2005) Standardisation of spirometry. Eur Respir J 26: 319-338.

9. The World Anti Doping Agency (2010) The International Standards for Laboratories Technical Document 2010: Decision limits for the confirmatory quantification of threshold substances.

10. Pluim B, de Hon O, Staal J, Limpens J, Kuipers H, et al. (2011) $\beta_{2}$-Agonists and physical performance: a systematic review and meta-analysis of randomized controlled trials. Sports Med. 41: 39-57.

11. Dickinson J, Hu J, Chester N, Loosemore M, Whyte G (2014) Acute impact of inhaled short acting b2-agonists on $5 \mathrm{~km}$ running performance. J Sports Sci Med 13: 271-279.

12. Elers J, Mørkeberg J, Jansen T, Belhage B, Backer (2012) High-dose inhaled salbutamol has no acute effects on aerobic capacity or oxygen uptake kinetics in healthy trained men. Scand J Med Sci Sports 22: 232-239.

13. Dickinson J, Molphy J, Chester N, Loosemore M, Whyte G (2014) The Ergogenic Effect of Long-Term Use of High Dose Salbutamol. Clin J Sport Med. 24:474-81.

14. Kalsen A, Hostrup M, Bangsbo J, Backer V (2014) Combined inhalation of beta2 -agonists improves swim ergometer sprint performance but not highintensity swim performance. Scand J Med Sci Sports 24: 814-22.

15. Hostrup M, Kalsen A, Auchenberg M, Bangsbo J, Backer V (2014) Effects of acute and 2-week administration of oral salbutamol on exercise performance and muscle strength in athletes. Scand J Med Sci Sports.

16. Pichon A, Venisse N, Krupka E, Perault-Pochat M, Denjean A (2006) Urinary and blood concentrations of $\beta 2$-agonists in trained subjects: comparison between routes of use. Int J Sports Med. 27: 187-192.

17. Elers J, Pedersen L, Henninge J, Hemmersbach P, Dalhoff K, et al. (2012) The pharmacokinetic profile of inhaled and oral salbutamol in elite athletes with asthma and nonasthmatic subjects. Clin J sports Med 22: 140-145.

18. Sporer B, Sheel W, McKenzie D (2008) Dose response of inhaled salbutamol on exercise performance and urine concentrations. Med Sci Sports Exerc 40: 149-157.

19. Elers J, Pedersen L, Henninge J, Hemmersbach, Dalhoff K, et al. (2011) Urine concentrations of repetitive doses of inhaled salbutamol. Int J Sports Med 32 574-579.

20. Parsons J, Hallstrand T, Mastronarde J, Kaminsky D, Rundell K, et al. (2013) An official American Thoracic Society clinical practice guideline: exercise-induced bronchoconstriction. Am J Respir Crit Care Med 187: 1016-1027.

This article was originally published in a special issue, entitled: "Sport Medicine and Exercise Physiology", Edited by Prof. Julien Baker, University of the West of Scotland, UK 